Sādhanā Vol. 39, Part 5, October 2014, pp. 1141-1150. (C) Indian Academy of Sciences

\title{
A novel approach for classification of abnormalities in digitized mammograms
}

\author{
S SHANTHI $^{1, *}$ and V MURALI BHASKARAN ${ }^{2}$ \\ ${ }^{1}$ Kongu Engineering College, Erode 638 052, India \\ ${ }^{2}$ Dhirajlal Gandhi College of Technology, Salem 636 309, India \\ e-mail: shanthi.kongumca@gmail.com; murali66@gmail.com
}

MS received 24 February 2013; revised 13 December 2013; accepted 17 January 2014

\begin{abstract}
Feature extraction is an important process for the overall system performance in classification. The objective of this article is to reveal the effectiveness of texture feature analysis for detecting the abnormalities in digitized mammograms using Self Adaptive Resource Allocation Network (SRAN) classifier. Thus, we proposed a feature set based on Gabor filters, fractal analysis, multiscale surrounding region dependence method (MSRDM) to identify the most common appearance of breast cancer namely microcalcification, masses and architectural distortion. The results of the experiments indicate that the proposed features with SRAN classifier can improve the classification performance. The SRAN classifier produces the classification accuracy of $98.44 \%$ for the proposed features with 192 images from MIAS dataset.
\end{abstract}

Keywords. MSRDM; Gabor features; fractal analysis; feature extraction; mammogram; SRAN.

\section{Introduction}

Breast cancer is by far the most prevalent cancer diagnosed in women worldwide. The breast cancer incident has increased in most countries worldwide in the last decades, with the most rapid increases occurring in many of the developing countries (International Agency for Research on Cancer 2008). In many African and Asian countries including India, breast cancer incidence and mortality rates have been raising (Jemal et al 2011). Until now there is no known way to avert breast cancer but the earlier the cancer can be detected, the higher the chance of survival for patients. At present, digital mammography is one of the most appropriate methods for early detection of breast cancer (Kuhl et al 2005). The most common mammographic signs of breast cancer are microcalcification, masses, architectural distortion and bilateral asymmetry (American College of Radiology 1998). Nowadays, vast amounts of mammograms are generated daily in hospitals and medical centers. Thus, the radiologists have more images to manually

${ }^{*}$ For correspondence 
analyse and the process of diagnosing them becomes tedious, and consequently more susceptible to errors.

Currently, Computer Aided Diagnosis (CAD) system could offer a cost effective alternative to double reading of mammograms and can suggest the radiologist about where the abnormalities present in the mammogram and what types of abnormalities present in it for reducing the errors in diagnosis (Paradkar \& Pande 2011; Karahaliou et al 2008; Mencattini et al 2010; Banik et al 2013; Rangayyan et al 2007; Shanthi \& Murali Bhaskaran 2012, 2013; Tiedeu et al 2012; Wei et al 2005; Oliver et al 2012; Matsubara et al 2000).

Calcifications are small calcium deposits that form in the breast because of benign or malignant processes. Mammographically, they appear as bright white spots of various sizes and shapes. The important characteristics of calcifications are their size, shape or morphology, number and distribution. Karahaliou et al (2008) developed a system for breast cancer diagnosis with microcalcifications based on the features such as Gray-level texture, Laws' texture energy measures and wavelet coefficient texture features. In this system, probabilistic neural network is used for classifying the mammogram and achieved area under Receiver Operating Characteristic (ROC) curve $(A z)$ of 0.989 for 85 mammographic images. The limitation of this work is that the training and test samples are selected by leave-one-out methodology. Therefore, the correlation between the data of the same patient may have favourably biased the reported classification performance. In addition, the system is evaluated with limited size of data set.

A 'Mass' is a space-occupying lesion seen in two different projections. If a potential mass is seen in only a single projection it should be called a 'Density' until its three-dimensionality is confirmed (ACR - BIRADS 1998). Radiologists characterize masses by their shape and margin properties. Mencattini et al (2010) presented a CADx system in which morphological, textural features based on gray level coocurrence matrix are extracted from regions of interest (ROIs), and Monte Carlo simulation is employed to classify the ROI as mass or normal tissue. For this experiment only sixteen mammographic images from the DDSM database are used.

Architectural distortion is the third most common mammographic sign of non-palpable breast cancer, and is defined by American College of Radiology (1998) in BI-RADS as follows. 'The normal architecture (of the breast) is distorted with no definite mass visible. This includes spiculations radiating from a point and focal retraction or distortion at the edge of the parenchyma. Architectural distortion can also be an associated finding'. Banik et al (2013) used Gabor filters, phase portrait analysis, analysis of the angular spread of power, fractal analysis, Laws' texture energy measures, and Haralick's texture features to detect the sites of architectural distortion in prior mammograms of interval-cancer cases. Analysis of the performance of the methods with free-response receiver operating characteristics indicated sensitivity of 0.80 and 0.90 at 5.8 and 8.1 false positives per image, respectively, with the Bayesian classifier and the leave-one-image-out method.

Asymmetry of breast parenchyma between the two sides has been one of the most useful signs for detecting primary breast cancer. Bilateral asymmetry, i.e., asymmetry of the breast parenchyma between left and right breast, may indicate breast cancer in its early stage. Rangayyan et al (2007) proposed a technique to analyse bilateral asymmetry in mammograms by combining directional information, morphological measures, and geometric moments related to density distributions. Eighty-eight mammograms from the Mini-MIAS database were used and achieved the classification accuracies of $84.4 \%$ with sensitivity and specificity rates of $82.6 \%$ and $86.4 \%$, respectively.

Several CAD systems have been reported on the detection and classification of microcalcification, masses, architectural distortion and bilateral asymmetry (Tang et al 2009). Although significant progress has been made over the last 20 years, much work still needs to be done to 
improve the accuracy of the CAD systems. Even though several methods of tissue identification are available in literature, not a single method is applicable for identification of all the signs of breast cancer (Tiedeu et al 2012; Wei et al 2005; Oliver et al 2012; Matsubara et al 2000; Nemoto et al 2009). Hence,we proposed the combination of features to detect microcalcification, masses and architectural distortion. In order to improve the classification performance, we present a system for the detection of abnormalities in the mammograms based upon Gabor filters, fractal analysis (Banik et al 2013), discrete wavelet transformation (Shanthi \& Murali Bhaskaran 2012), multiscale surrounding region dependence method (Shanthi \& Murali Bhaskaran 2013) and SRAN (Suresh et al 2010) classifier.

The remaining part of the article is organized as follows. Section 2 gives the details of ROI identification. Section 3 discusses the feature extraction techniques. Section 4 describes SRAN concepts. Section 5 illustrates the experimental results. Finally, section 6 provides the conclusion.

\section{Identification of ROI}

The mammogram images used in our experiments were taken from the Mammographic Image Analysis Society (MIAS). For each abnormal case, the coordinates of center of abnormality is provided along with the approximate radius (in pixels) of a circle enclosing the abnormality. Knowing the location and the approximate size of abnormality allows us to extract ROI with proper dimension representing the tumour zone.

\section{Feature extraction}

Critical issue in CAD system is the extraction/selection of the best set of features for detecting or classifying the suspect lesions. In the present work, features computed from Gabor filters (Banik et al 2013), fractal analysis (Chaudhuri \& Sarkar 1995), multiscale surrounding region dependence method (Shanthi \& Murali Bhaskaran 2013) are used to classify the ROI into normal or benign or malignant.

\subsection{Multi resolution analysis - Discrete wavelet transform}

Multiresolution analysis has proved to be useful in mammographic image processing, image enhancement and feature extraction. The common task is to decompose the original image into subbands that preserve high and low frequency information. Several studies investigated the use of Discrete Wavelet Transform (DWT) as a multiresolution analysis tool for feature analysis and classification. Two-dimensional discrete wavelet transform is computed by applying a separable filter bank to the image as defined in Eqs. (1)-(4).

$$
\begin{aligned}
L_{n}\left(b_{i}, b_{j}\right) & =\left[H_{x} *\left[H_{y} * L_{n-1}\right]_{\downarrow 2,1}\right]_{\downarrow 1,2}\left(b_{i}, b_{j}\right), \\
D_{n 1}\left(b_{i}, b_{j}\right) & =\left[H_{x} *\left[G_{y} * L_{n-1}\right]_{\downarrow 2,1}\right]_{\downarrow 1,2}\left(b_{i}, b_{j}\right), \\
D_{n 2}\left(b_{i}, b_{j}\right) & =\left[G_{x} *\left[H_{y} * L_{n-1}\right]_{\downarrow 2,1}\right]_{\downarrow 1,2}\left(b_{i}, b_{j}\right), \\
D_{n 3}\left(b_{i}, b_{j}\right) & =\left[G_{x} *\left[G_{y} * L_{n-1}\right]_{\downarrow 2,1}\right]_{\downarrow 1,2}\left(b_{i}, b_{j}\right),
\end{aligned}
$$


where $*$ denotes the convolution operator, $\downarrow_{2,1}$ subsampling along the rows, $\downarrow_{1,2}$ subsampling along the columns and $L_{0}=I(\vec{x})$ is the original image. $\mathrm{H}$ and $\mathrm{G}$ are a low and bandpass filter, respectively. $L_{n}$ is obtained by low pass filtering and is therefore referred to as the low resolution image at scale n. The detail images $D_{n i}$ are obtained by bandpass filtering in a specific direction and they contain directional detail information at scale $\mathrm{n}$. The original image $\mathrm{I}$ is thus represented by a set of subimages at several scales: $\left\{L_{d}, D_{n i}\right\}_{i=1,2,3 ; n=1,2 . . d}$ This is a multiscale representation of depth $\mathrm{d}$ of the image $\mathrm{I}$.

\subsection{MSRDM}

For each ROI, the 2D-DWT is applied using the Db3 wavelet and obtained three detail coefficient matrices and one approximation coefficient matrix. During various levels of decomposition, the multiscale surrounding region dependence matrices are computed from each subbands (Shanthi $\&$ Murali Bhaskaran 2013). Consider three square windows $w_{1}, w_{2}$ and $w_{3}$ centred at a current pixel $(\mathrm{x}, \mathrm{y})$ of the decomposed image as in figure 1 .

Where $R_{1}$ is the inner surrounding region and $R_{2}$ is the outer surrounding region, and $w_{1}, w_{2}$ and $w_{3}$ denote the sizes of each square region. Then, the surrounding region dependence matrix is obtained for each subband using Eq. (5).

$$
M(q)=[\propto(i, j)], 0 \leq i \leq m, 0 \leq j \leq n,
$$

where $q$ is the given threshold value, and the values of $m$ and $n$ are the total numbers of pixels of regions $R_{1}$ and $R_{2}$, respectively. The elements of $\alpha(\mathrm{i}, \mathrm{j})$ is defined as in Eq. (6).

$$
\propto(i, j)=\#\left\{(x, y)\left|c R_{1}(x, y)=i,(x, y)\right| c R_{2}(x, y)=j,(x, y) \in L_{x} X L_{y}\right\},
$$

where \# represents the number of elements in the set, and $L_{x} \times L_{y}$ is the 2-D image space. In Eq. (6), $c R_{1}(\mathrm{x}, \mathrm{y})$ and $c R_{2}(\mathrm{x}, \mathrm{y})$ are the inner and outer count of the current pixel (x,y) as given in Eqs. (7) and (8), respectively.

$$
\begin{aligned}
& c R_{1}(x, y)=\#\left\{(k, l) \mid(k, l) \in R_{1} \quad \text { and }[I(x, y)-I(k, l)]>q\right\}, \\
& c R_{2}(x, y)=\#\left\{(k, l) \mid(k, l) \in R_{2} \quad \text { and }[I(x, y)-I(k, l)]>q\right\},
\end{aligned}
$$

where $\mathrm{I}(\mathrm{x}, \mathrm{y})$ is the image intensity of the current pixel $(\mathrm{x}, \mathrm{y})$. The threshold value $\mathrm{q}$ is obtained as 80 by experiments. The window size of $w_{1}, w_{2}, w_{3}$ are 7,5 and 3 , respectively.

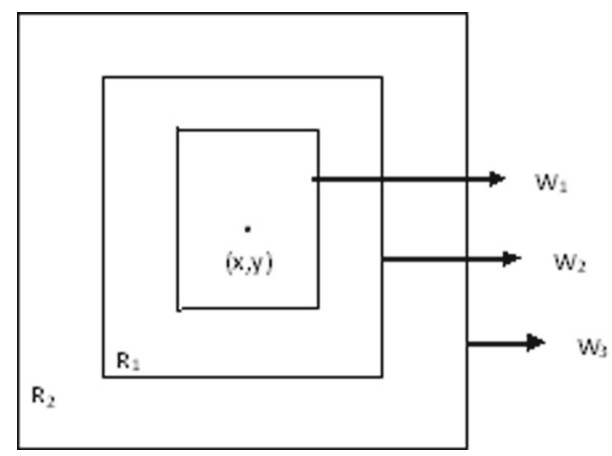

Figure 1. Configuration of the surrounding regions on the current pixel $(\mathrm{x}, \mathrm{y})$. 
From the multiscale surrounding region dependence matrix, the Horizontal Weighted Sum (HWS), Vertical Weighted Sum (VWS), Diagonal Weighted Sum (DWS) and Grid Weighted Sum (GWS) features are calculated by using the Eqs. (9)-(12).

$$
\begin{gathered}
H W S=\frac{1}{N} \sum_{i=0}^{m} \sum_{j=0}^{n} j^{2} r(i, j), \\
V W S=\frac{1}{N} \sum_{i=0}^{m} \sum_{j=0}^{n} r^{2} r(i, j), \\
D W S=\frac{1}{N} \sum_{k=0}^{m+n} k^{2}\left[\sum_{i=0}^{m} \sum_{j=0}^{n} r(i, j)\right]_{i+j=k}, \\
G W S=\frac{1}{N} \sum_{i=0}^{m} \sum_{j=0}^{n} i j r(i, j),
\end{gathered}
$$

where $N$ is the total sum of elements in the surrounding region dependence matrix,

$$
N=\sum_{i=0}^{m} \sum_{j=0}^{n} \propto(i, j)
$$

and $r(i, j)$ is the reciprocal of the element, which is defined as

$$
r(i, j)=\left\{\begin{array}{cl}
\frac{1}{\propto(i, j)}, & \text { if } \propto(i, j)>0 \\
0, & \text { Otherwise }
\end{array}\right.
$$

\subsection{Fractal analysis}

Fractal analysis provides a powerful tool in many medical imaging applications because of the self-similarity property (Lopes \& Betrouni 2009). Fractal Dimension (FD) is a real number describes the fractal property of the object. There are several different methods to calculate FD, such as box counting, differential box counting, extended counting method, triangular prisms method, power spectrum, Isarithm method (Lopes \& Betrouni 2009). The presence of architectural distortion disrupts the self-similarity properties, and thereby changes the fractal dimension of breast parenchyma. Hence, FD can be used as a discriminating feature to distinquish normal and abnormal with architectural distortion. The differential box counting method was chosen for its simplicity and efficiency. The differential box counting method work on grey-scale image and thus the conversion step is avoided (Chaudhuri \& Sarkar 1995). By covering the ROI with boxes of size s, the FD is calculated as

$$
F D=\lim _{r \rightarrow 0} \frac{\log (N(s))}{\log (s)},
$$

where $N(s)$ is the difference between the minimum and the maximum grey levels in the $(i, j)^{\text {th }}$ box. Lacunarity is a counterpart to the fractal dimension that describes the texture of a fractal. If a fractal has large gaps or holes, it has high lacunarity; on the other hand, if a fractal is almost 
translationally invariant, it has low lacunarity. Different fractals can be constructed that have the same dimension but that look widely different because they have different lacunarity. FD and lacunarity are calculated for each ROI.

\subsection{Gabor filter}

Gabor filters are similar to those of the human visual system, and they found to be particularly appropriate for texture representation and discrimination. The Gabor filters are self-similar. A set of Gabor filters with different frequencies and orientations may be helpful for extracting useful features from an image. In the spatial domain, a 2D Gabor filter can be viewed as a Gaussian kernel function modulated by a sinusoidal plane of particular frequency and orientation. It can be written as

$$
f(x, y)=\frac{1}{2 \Pi \sigma_{x} \sigma_{y}} \exp \left[\left[-\frac{1}{2}\left(\frac{x^{2}}{\sigma_{x}^{2}}+\frac{y^{2}}{\sigma_{y}^{2}}\right)\right]+(2 \Pi j u x)\right],
$$

where, $\mathrm{u}$ denotes the radial frequency of the Gabor function. The constants $\sigma_{x}$ and $\sigma_{y}$ define the spread of Gabor envelop along $x$ and y-axes. After applying Gabor filters on the image with different orientation at different scale, we obtain an array of magnitudes. These magnitudes represent the energy content at different scale and orientation of the image. Sixteen Gabor filters are generated at 4 scales $(2,4,32,64)$ and 4 orientations (horizontal, vertical, 45 and 135). Therefore, 16 sub images obtained from Gabor filter bank are considered for computation of energy. The Gabor energy is closely related to the local power spectrum. The local power spectrum associated with pixel in an image is defined as the squared modulus of the Fourier transform of the product between the image and a window function. This window function has the role of choosing a neighbourhood pixel of interest.

$$
\text { Energy }=\Sigma \Sigma\left\|M_{p q}(x, y)\right\|^{2} .
$$

\section{Self adaptive resource allocation network (SRAN) classifier}

The SRAN classifier is a sequential learning algorithm with self-regulated control parameters. Since, the SRAN classifier uses explicit classification error in growing/learning criterion and discarding similar samples, it prevents overtraining and provides better generalization performance (Suresh et al 2010). Assume that, there are observation data $\left\{\left(x_{1}, y_{1}\right),\left(x_{2}, y_{2}\right), \ldots\left(x_{\mathrm{t}}, y_{\mathrm{t}}\right), ..\right\}$ where $x_{t} \in \mathcal{R}^{m}$ is an m-dimensional features of observation $\mathrm{t}$ and $y_{t} \in \mathcal{R}^{n}$ is its coded class label. Here, $\mathrm{n}$ represents the total number of classes. If the feature observation $\mathrm{x}$ is assigned to the class label $\mathrm{c}$, then $\mathrm{c}^{\text {th }}$ element of $\mathrm{y}=\left[\mathrm{y}_{1}, \mathrm{y}_{2} \ldots \mathrm{y}_{\mathrm{c}}, \ldots \mathrm{y}_{\mathrm{n}}\right]^{\mathrm{T}}$ is 1 and other elements are -1 .

In the SRAN system, the training sample record arrives one by one and the network adapts its parameters on the basis of the difference in knowledge between the network and the current sample record. The maximum error $\mathrm{E}$ is calculated by using hinge loss function as defined in Eq. (18).

$$
\begin{gathered}
\mathrm{E}=\max _{\mathrm{i}=1,2,3 \ldots \mathrm{n}}\left|\mathrm{e}_{\mathrm{i}}\right| \\
e_{i}=\left\{\begin{array}{cc}
\mathrm{y}_{\mathrm{i}}-\mathrm{y}_{\mathrm{i}} & \text { if } \mathrm{y}_{\mathrm{i}} \dot{y}_{\mathrm{i}}<1 \\
0 & \text { Otherwise }
\end{array} \quad \mathrm{i}=1,2, \ldots \mathrm{n},\right.
\end{gathered}
$$


where $y_{i}$ is the actual class label of $i^{\text {th }}$ sample and $y_{i}$ is the predicted class label of $i^{\text {th }}$ sample.

The output of this classifier is defined with k hidden neurons as defined in Eq. (20).

$$
\dot{y}_{i}=\sum_{j=1}^{k} \propto_{i j} y_{h}^{j} \quad i=1,2, \ldots n,
$$

where $\alpha_{\mathrm{ij}}$ is the weight connecting the $\mathrm{i}^{\text {th }}$ output neuron and $\mathrm{j}^{\mathrm{th}}$ Gaussian neuron and $\mathrm{y}_{\mathrm{h}}^{\mathrm{j}}$ Gaussian basis function as defined in Eq. (21).

$$
\mathrm{y}_{\mathrm{h}}^{\mathrm{j}}=\exp \left(-\frac{\left\|\mathrm{x}-\mu_{\mathrm{j}}^{1}\right\|^{2}}{\left(\sigma_{\mathrm{j}}^{1}\right)^{2}}\right),
$$

where $\mu_{\mathrm{j}}^{1}$ and $\sigma_{\mathrm{j}}^{1}$ are the mean and standard deviation, respectively, and $\left\|\_\right\|$denotes the Euclidean norm. As each new sample is presented to the network, according to the sample error the self-regulatory system would perform any one of the following three actions.

(1) The sample is used for:

a) Network growing: if the predicted and actual class label is not the same and the error $\mathrm{E}$ is greater than self-regulated control parameter $\left(\eta_{\mathrm{g}}\right)$, then the sample is to be used to add a new hidden neuron in the network.

$$
\hat{\mathrm{c}} \neq \mathrm{c} \text { and } \mathrm{E} \geq \eta_{\mathrm{g}} .
$$

b) Network learning: if the predicted and actual class label is same and the error E is greater than the self-regulated learning control parameter $\left(\eta_{1}\right)$, then the network parameters are updated.

$$
\hat{\mathrm{c}}=\mathrm{c} \text { and } \mathrm{E} \geq \eta_{1} .
$$

The SRAN classifier uses the extended Kalman filter (EKF) to update the network parameters as defined in Eq. (24).

$$
\mathrm{W}_{(\mathrm{t})}=\mathrm{W}_{(\mathrm{t}-1)}+\mathrm{KL}_{(\mathrm{t})} \mathrm{e}
$$

where $\mathrm{KL}(\mathrm{t})$ is the Kalman gain and e is the error obtained from hinge loss function. The self-regulated control parameters $\left(\eta_{1}, \eta_{\mathrm{g}}\right)$ are updated using the Eqs. (25) and (26).

$$
\begin{gathered}
\eta_{1}=\delta \eta_{1}-(1-\delta) \mathrm{E}, \\
\eta_{\mathrm{g}}=\delta \eta_{\mathrm{g}}-(1-\delta) \mathrm{E},
\end{gathered}
$$

where $\delta$ is a parameter that controls the slope of the decrease of the control parameter which is close to one.

(2) If Eqs. (22) and (23) are not satisfied then the sample has moved to the rear end of the stack for learning in future.

(3) If the sample error $\mathrm{E}$ is less than 0.05 , the sample has deleted from the dataset without using as a training sample to construct the network and thus it prevents over-training. 


\section{Experimental results and discussion}

The data set used in the current experiment has been taken from the Mammographic Image Analysis Society (Suckling et al 1994). Its corpus consists of 322 images belonging to three big categories: normal, benign and malign. The benign and malign which indicate different signs of abnormalities such as calcification (CALC), well-defined circumscribed masses (CIRC), speculated masses (SPIC), ill-defined masses (MISC), architectural distortion (ARCH), asymmetry (ASYM). In this work, we have considered only microcalcification, mass and architectural distortion. Therefore, we have selected the images with microcalcification, mass and architectural distortion along with the 100 normal images to have an equal proportion of normal and abnormal images. Figures $2 \mathrm{a}-\mathrm{b}$ show the different signs in benign and malignant categories from MIAS data set and samples selected from MIAS database for the current study, respectively.

To evaluate the discriminating abilities of the feature set, the C4.5, RBFN and SRAN classifiers have been employed. The dataset is divided into k subsets, and the holdout method is repeated $\mathrm{k}$ times. Each time, one of the $\mathrm{k}$ subsets is used as the test set and the other $\mathrm{k}-1$ subsets are put together to form a training set. Then the average performance across all $\mathrm{k}$ trials is computed. Every data point appears in a test set exactly once, and appears in a training set k-1 times. In this study the value of $\mathrm{k}$ is 10 .

Table 1 summarizes the classification accuracy of the existing features and the classification accuracy of the proposed integrated features using SRAN classifier.

Note that each approach used a different set of images coming also from different databases with different signs of abnormalities and hence the comparison has been done only in a qualitative way. Examination of table 1 indicates that the performance of the proposed technique is comparable and better than that achieved by a number of existing techniques. A direct comparison with our earlier work on analysis of microcalcification and mass (Shanthi \& Murali Bhaskaran 2013) indicates that the proposed technique has performed better on the dataset.

The principle of a good discriminating feature is more correlated with decision attribute and uncorrelated with other conditional attributes. The MSRDM features highly correlated with decision attributes and uncorrelated with the conditional attributes (Shanthi \& Murali Bhaskaran 2013). Cancerous tumours demonstrate a certain degree of randomness related with their growth, and are naturally asymmetrical and intricate in shape; therefore, FD can provide a better measure of their complex patterns. The energy is computed at each pixel for each combination of wavelength and orientation; the energy is the sum, over the phases, of the squared convolution values.

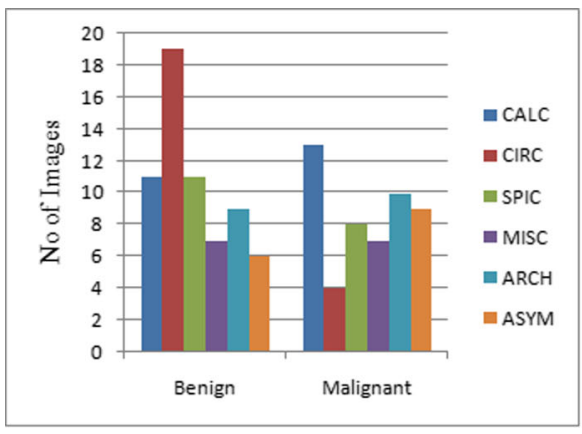

(a)

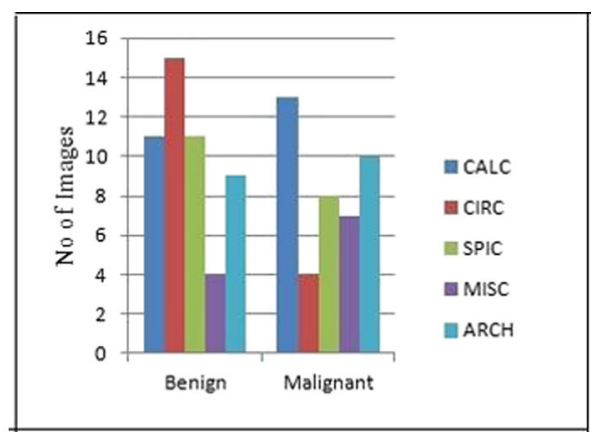

(b)

Figure 2. The distribution of abnormalities in benign and malignant categories. (a) MIAS data set. (b) Samples used in the current work from MIAS data set. 
Table 1. Summary of classification performance with existing techniques and proposed features.

\begin{tabular}{lcccc}
\hline & Signs of breast & \multicolumn{3}{c}{ Accuracy } \\
\cline { 3 - 5 } Method & cancer & MIAS & DDSM & Real data set \\
\hline Banik et al 2013 & ARCH & - & - & ROC of 0.75 \\
Biswas 2011 & ARCH & Sensitivity of 81.3 & & - \\
Karahaliou et al 2008 & CALC & - & AUC of 0.989 & - \\
Matsubara et al 2000 & ARCH & - & - & $94 \%$ \\
& & & & $84 \%$ \\
Oliver et al 2012 & CALC & ROC of .903 & ROC of .918 & - \\
Shanthi \& Murali Bhaskaran 2012 & CALC, Mass & $92.3 \%$ & & $98.3 \%$ \\
Shanthi \& Murali Bhaskaran 2013 & CALC, Mass & $84.29 \%$ & & $89 \%$ \\
Tiedeu et al 2012 & CALC & - & - & $\mathrm{A}_{z}$ of .085 \\
Wei et al 2005 & CALC & - & - & - \\
Proposed method & CALC, Mass, ARCH & $\mathbf{9 8 . 4 4 \%}$ & - & \\
\hline
\end{tabular}

Table 2. Number of samples used by different classifiers for training the model.

\begin{tabular}{lc}
\hline Classifiers & No. of samples used by the classifier for training \\
\hline C4.5 & 192 \\
RBFN & 192 \\
SRAN & 180 \\
\hline
\end{tabular}

Therefore, the proposed combination of MSRDM, FD and Gabor energy features outperforms the other features.

From table 2 we can observe that the SRAN classifier used only 180 samples for training the model while C4.5 and RBFN classifiers used the entire 192 samples for generating best classifier.

In standard online/sequential learning, the samples are presented only once, and all the samples are learnt and the arrival of similar samples leads to over-training of particular pattern. In SRAN, the sequence of the training sample is controlled internally using self-regulated control parameters. The sample without significant information is removed from the training set. Hence, this avoids over-training, reduces learning time, and minimizes the computational effort. Therefore, the SRAN classifier generates the best sequence of training samples from a given training sequence such that the classifier produces better generalization performance (Suresh et al 2010).

\section{Conclusion}

The advantage of this approach lies in the fact that it concentrates on three types of abnormalities. In addition, the SRAN classifier removes the training samples, which are similar to the knowledge already stored in the model. Therefore, it avoids the overtraining and reduces the learning time. Although by now some progress has been achieved, there are remaining challenges and directions for future research, such as

- Better enhancement and segmentation algorithms are required.

- Defining a standard test set and better evaluation criteria are still very important.

Further studies can be conducted with large number of real databases in a clinical environment. 


\section{References}

American College of Radiology (ACR) 1998 Illustrated breast imaging reporting and data system (BI-RADS). Am. College of Radiology, Reston, VA, 3rd edition

Banik S, Rangayyan R M and Desautels J E L 2013 Measures of angular spread and entropy for the detection of architectural distortion in prior mammograms. Int. J. Comput. Assist. Radiol. Surg. 8: $121-134$

Biswas S K 2011 Recognizing architectural distortion in mammogram: A multiscale Texture Modeling Approach with GMM. IEEE Trans. Biomed. Eng. 58(7): 2023-2030

Chaudhuri B and Sarkar N 1995 Texture segmentation using fractal dimension. IEEE Trans. Pattern Anal. Mach. Intell. 17(1): 72-77

International Agency for Research on Cancer (IARC) 2008 World Cancer Report 2008: IARC

Jemal A, Bray F, Center M M, Ferlay J, Ward E and Forman D 2011 Global cancer statistics. CA Cancer J. Clin. 61(2): 69-90

Karahaliou A N et al 2008 Breast cancer diagnosis: Analyzing texture of tissue surrounding microcalcifications. IEEE Trans. Inf. Technol. Biomed. 12(6): 731-738

Kuhl C K, Schrading S, Leutner C C, Morakkabati-Spitz N, Wardelmann E, Fimmers R, Kuhn W and Schild H H 2005 Mammography, breast ultrasound, and magnetic resonance imaging for surveillance of women at high familial risk for breast cancer. J. Clin. Oncol. 23(33): 8469-8476

Lopes R and Betrouni N 2009 Fractal and multifractal analysis: A review. Med. Image Anal. 13: 634-649

Matsubara T, Yamazaki D, Fujita H, Hara T, Iwase T and Endo T 2000 An automated classification method for mammograms based on evaluation of fibroglandular breast tissue density in Proc. 5th International Workshop on Digital Mammography, Yaffe M J (Ed.) Toronto, Ontario, Canada, 2000, 737-741

Mencattini A, Salmeri M, Rabottino G and Salicone S 2010 Metrological characterization of a CADx system for the classification of breast masses in mammograms. IEEE Trans. Instrum. Meas. 59(11): 2792-2799

Nemoto M, Honmura S, Shimizu A, Furukawa D, Kobatake H and Nawano S 2009 A pilot study of architectural distortion detection in mammograms based on characteristics of line shadows. Int. J. Comput. Assist. Radiol. Surg. 4(1): 27-36

Oliver A, Torrent A, Llado X, Tortajada M, Tortajada L, Sentis M, Freixenet J and Zwiggelaar R 2012 Automatic microcalcification and cluster detection for digital and digitised mammograms. Knowl-Based System 28: 68-75

Paradkar S and Pande S S 2011 Intelligent detection of microcalcification from digitized mammograms. Sadhana - Acad. Proc. Eng. Sci. 36(1): 125-139

Rangayyan R M, Ferrari R J and Frere A F 2007 Analysis of bilateral asymmetry in mammograms using directional, morphological, and density features. SPIE Proc. J. Electronic Imaging 16(01): 013003-1013003-12

Shanthi S and Murali Bhaskaran V 2012 Computer aided system for detection and classification of breast cancer. Int. J. Inf. Technol. Control Autom. 2(4): 87-98

Shanthi S and Murali Bhaskaran V 2013 A novel approach for detecting and classifying breast cancer in mammogram images. Int. J. Intell. Inf. Technol. 9(1): 21-39

Suckling J et al 1994 The mammographic image analysis society digital mammogram database. Proc. Int. Workshop Dig. Mammography, 1069: 375-378

Suresh S, Dong K and Kim H J 2010 A sequential learning algorithm for self-adaptive resource allocation network classifier. Neurocomputing 73(16-18): 3012-3019

Tang J, Rangayyan R M, Xu J, Naqa I EI and Yang Y 2009 Computer-aided detection and diagnosis of breast cancer with mammography: Recent advances. IEEE Trans. Inf. Technol. Biomed. 13(2): 236-251

Tiedeu A, Daul C, Kentsop A, Graebling P and Wolf D 2012 Texture-based analysis of clustered microcalcifications detected on mammograms. Digit. Signal Process. 22: 124-132

Wei L, Yang Y, Nishikawa R M and Jiang Y 2005 A study on several machine-learning methods for classification of malignant and benign clustered microcalcifications. IEEE Trans. Med. Imaging 24(3): $371-380$ 